\title{
EPIMORPHISMS AND SEMIGROUP VARIETIES
}

\author{
Peter M. Higgins
}

Most of the new material in this thesis appears elsewhere in papers by the author. The new material in each chapter can be found, in the main, in the papers immediately following its description.

This thesis is concerned with three related questions.

1. What semigroup varieties are epimorphically closed?

2. What semigroup varieties are saturated?

3. What semigroup varieties are absolutely closed?

Chapter 1 contains introductory material including a proof of Isbell's Zigzag Theorem on which the thesis is based.

In the second chapter we prove that epis are onto for generalized inverse semigroups (see [1]).

In Chapter 3 we show that the absolutely closed varieties are those consisting entirely of semilattices of groups, or of right groups, or of left groups (see [2]).

In Chapter 4 we determine all saturated varieties of commutative semigroups in a joint theorem with N.M. Khan. They are the varieties admitting a non-trivial identity for which at least one side contains no repeated variable. This result is refined to give a five-way characterization of these varieties, which includes their determination as the commutative varieties consisting entirely of nilpotent extensions of semilattices of

Received 28 March 1984. Thesis submitted to Monash University, August 1983. Degree approved December 1983. Supervisor: Dr T.E. Hall.

Copyright Clearance Centre, Inc. Serial-fee code: 0004-9727/84 $\$$ A2.00 +0.00 .

415 
groups. The details are in [3].

In the fifth chapter we derive necessary conditions for a variety to be saturated, and for a variety to be epimorphically closed.

In Chapter 6 we give a survey of various nil conditions, and then prove that several types of nil extensions of saturated semigroups are themselves saturated. Their first application is in proving the five-way characterization of the saturated heterotypical varieties, which includes their realization as the varieties consisting entirely of nilpotent extensions of completely simple semigroups.

Theorem 7.1 gives a sufficient condition for a variety to be saturated, which is generalized in 7.12. The proof of 7.1 is structurally based, and forms the pattern for 7.3 and 7.5 , in which we characterize the (saturated) varieties consisting entirely of nilpotent extensions of semilattices of groups, and of generalized inverse semigroups respectively. The reader is referred to [5] for more information on Chapters 5 and 6 and Section 1 of Chapter 7. Section 2 of Chapter 7 gives an example of a band which is not saturated. This answers in the negative a question posed by Hall: "Are all regular semigroups saturated?" (See [4] for details.)

In Chapter 8 , Section 1 , the permutation identities which ensure that a semigroup variety is epimorphically closed are determined in a joint theorem with N.M. Khan (see [6]). In the second section the permutation identities that ensure a semigroup variety is finitely based are determined (see [7]).

The motivating question in the ninth chapter is: "What identities ensure that a finite semigroup is saturated?" Using an example of a nonsaturated finite semigroup we get a partial answer. The question is answered affirmatively for heterotypical and permutation identities: the latter assisting in the determination of all saturated semigroups up to order four (see [6]).

Chapter 10 is a list of questions which have arisen out of this research. 


\section{References}

[1] Peter M. Higgins, "Epis are onto for generalized inverse semigroups", Semigroup Formom 23 (1981), 255-259.

[2] Peter M. Higgins, "The determination of all absolutely closed varieties of semigroups", Proc. Amer. Math. Soc. 87 (1983), 419-421.

[3] Peter M. Higgins, "The commutative varieties of semigroups for which epis are onto", Proc. Edinburgh Math. Soc. Sect. A 94 (1983), $1-7$.

[4] Peter M. Higgins, "A semigroup with an epimorphically embedded subband", Bull. Austral. Math. Soc. 27 (1983), 231-242.

[5] Peter M. Higgins, "Saturated and epimorphically closed varieties of semigroups", J. Austral. Math. Soc. Ser. A 36 (1984), 153-175.

[6] Peter M. Higgins, "Epimorphisms, permutation identities and finite semigroups", Semigroup Forum (to appear).

[7] Peter M. Higgins, "The permutation identities which ensure that a semigroup variety is finitely based", submitted.

Department of Mathematics,

California State University at Chico,

Chico,

California 95929-0525,

USA. 